\title{
Reacciones adversas inmediatas a la donación: frecuencia y caracterización, Banco de Sangre de la Clínica Cardiovascular Santa María, Medellín, 1999
}

\author{
María I. Múnera ${ }^{1}$, Blanca S. Ramírez ${ }^{2}$, Claudia M. Zapata ${ }^{1}$, Dora S. Marín ${ }^{1}$ \\ 1 Banco de Sangre, Clínica Cardiovascular Santa María, Medellín, Colombia. \\ ${ }^{2}$ Facultad de Odontología, Universidad de Antioquia, Medellín, Colombia.
}

\begin{abstract}
Disponer de un flujo suficiente de donantes altruistas que permita la obtención de componentes sanguíneos de buena calidad es el problema más importante en los bancos de sangre en nuestro medio. La presencia de reacciones adversas a la donación puede influir en forma negativa en la disposición del donante para regresar al banco de sangre. Con el objetivo de establecer la frecuencia y explorar los factores de riesgo involucrados en la aparición de reacciones adversas a la donación, se realizó un estudio de corte, basado en los registros de donantes del Banco de Sangre de la Clínica Cardiovascular Santa María de Medellín durante 1999. La edad promedio de los donantes estudiados fue de 33 años en mujeres y 34 en hombres; se encontró una frecuencia de reacciones adversas de $2,7 \%$ y la predominante fue la reacción vasovagal con el 1,2\%. Los posibles factores de riesgo relacionados con las reacciones adversas, en general, fueron donación por primera vez, edad menor de 25 años y sexo femenino.
\end{abstract}

Palabras clave: donantes de sangre, reacciones adversas, reacciones vasovagales, factores de riesgo.

\section{Adverse reactions after donation: frequency and characterization, Santa Maria Cardiovascular Clinic Blood Bank, Medellín, Colombia, 1999}

A major problem facing clinical blood banks is the procurement of numbers of altruistic blood donors sufficient to guarantee the quality of blood components. Adverse reactions to donation can negatively influence altruistic donors for new donations. A cross sectional study was carried out at Santa María Cardiovascular Clinic in Medellín during 1999 to identify the frequency and risk factors involved in adverse reactions to donation. The donors mean age was 33 years in females and 34 in males. The frequency of adverse reactions was $2.7 \%$; the most important class was vasovagal reactions in $1.2 \%$. Risk factors related to adverse reactions were first-time donations, age below 25 years and femaleness.

Key words: blood donors, adverse reactions, vasovagal reactions, risk factors.

Las reacciones adversas a la donación ocurren en 2 a $5 \%$ de los donantes de sangre $(1,2)$; en general, se clasifican en aquéllas relacionadas con la venopunción, efectos vasovagales o hipotensión $(3,4)$. Los síntomas informados con mayor frecuencia incluyen debilidad, diaforesis, piel fría y palidez que, en la mayoría de los casos, se

Correspondencia:

M.I. Múnera, Banco de Sangre, Clínica Cardiovascular Santa María, Medellín, Colombia.

Recibido: 18/04/00; aceptado: 29/06/01 deben a reacciones vasovagales por el estrés sicológico de la situación o a factores neurológicos. Los bancos de sangre tienen la responsabilidad de establecer estrategias para prevenir y detectar precozmente la presencia de reacciones adversas en los donantes que acuden a sus instalaciones. Factores como el entrenamiento del personal, el trato dado al donante durante todo el proceso, el ambiente y la selección del sitio de la punción, entre otros, juegan un papel importante en la aparición de reacciones adversas. Con el objetivo de establecer la frecuencia e identificar las causas 
de las reacciones adversas a la donación, así como explorar los factores de riesgo involucrados, se realizó un estudio de corte en el Banco de Sangre de la Clínica Cardiovascular Santa María de Medellín durante 1999.

\section{Materiales y métodos}

Con base en la revisión de los registros de donación del Banco de Sangre de la Clínica Cardiovascular Santa María, correspondientes al período comprendido entre enero y diciembre de 1999, se hizo un estudio analítico de corte y retrospectivo.

Se revisaron 3.300 registros de todos los donantes que asistieron a la clínica durante el período en mención. Con el propósito de garantizar la confidencialidad, se identificaron los formatos por medio de códigos. Los datos se recolectaron de los registros de los donantes y se consignaron en un formato diseñado para tal fin; dicha información incluía variables sobre identificación, sexo, edad, peso, presión arterial sistólica y diastólica, niveles de hemoglobina, antecedentes de efectos adversos a la donación, antecedentes de donación previa, presencia y tipo de reacción luego de la donación.

Se consideraron como tipos de reacción adversa a la donación la presencia de dolor en el sitio de la punción, la aparición de hematoma, la donación lenta, la hiperventilación, la hipotensión y la reacción vasovagal.

Para la cuantificación de los niveles de hemoglobina, el laboratorio de la clínica utiliza el fotómetro de $\beta$-hemoglobina HemoCue ( ${ }^{\circledR}$ HemoCue Inc., Canadá); la determinación del peso corporal se hace en una báscula (Soehnle, Alemania) y para la evaluación de la presión arterial se utiliza un tensiómetro electrónico Dinamap XL (® Critikon, Tampa, USA).

Para el análisis de la información, se procesaron los datos en los programas estadísticos Epi-info 6.04 y SPSS 7.5. Se utilizaron medidas de estadística descriptiva tales como proporciones, promedios y medidas de variación; además, se hizo un análisis de corte para explorar la asociación entre la presencia de reacción y diferentes factores de riesgo, definidos como hemoglobina menor de $13 \mathrm{~g} / \mathrm{dl}$, peso menor de 55 $\mathrm{kg}$, edad menor de 25 años, presión sistólica $\leq 100$ $\mathrm{mm} \mathrm{Hg}$, presión diastólica $\leq 60 \mathrm{~mm} \mathrm{Hg}$ y ser primera donación. Para establecer si existían diferencias estadísticamente significativas según sexo, en los niveles de hemoglobina, edad, peso, y presión sistólica y diastólica, se hizo una prueba no paramétrica (Kruskal Wallis) debido a que se estableció que el grupo de estudio no seguía una distribución normal para dichas variables.

\section{Resultados}

El análisis de los resultados se hizo con base en los registros de 3.236 donantes, después de haber descartado 64 encuestas con datos incompletos.

La edad promedio del grupo de estudio fue de 33 años con una desviación estándar (DE) de 9,2 años. En la distribución según edad se observó la mayor proporción en el grupo de 30 a 39 años, seguido del grupo de 20 a 29.

La distribución porcentual según el sexo fue de $40,8 \%$ para mujeres y $59,2 \%$ para hombres. Además, el $42,6 \%$ fueron donantes de primera vez.

El peso promedio para el sexo masculino fue de $73 \mathrm{~kg}$ (DE +/- 11) y para el femenino, de $62 \mathrm{~kg}$ (DE +/-9). En el cuadro 1 se presentan los valores promedio para edad, peso, valores de hemoglobina, presión sistólica y presión diastólica. Del total de donantes estudiados, sólo uno informó sobre antecedentes de reacción adversa.

Hubo diferencias estadísticamente significativas según sexo en los valores promedio de hemoglobina, edad, presión diastólica, presión sistólica y peso (cuadro 1).

De los donantes evaluados, 90 (2,7\%) presentaron alguna reacción adversa. Del total de reacciones, la más frecuente fue la reacción vasovagal con el $43 \%$ y la menos frecuente fue el dolor en el sitio de la punción, con el 1,1\%. La distribución porcentual según el tipo de reacción se ilustra en la figura 1.

En la exploración de los factores de riesgo, se encontró un riesgo mayor de presentar reacción adversa a la donación en mujeres, en menores de 25 años y en donantes de primera vez (cuadro 2).

\section{Discusión}

Aunque las reacciones adversas a la donación no son de alta frecuencia, su ocurrencia tiene un efecto 
Cuadro 1. Valores promedio y desviación estándar según sexo, de las características estudiadas en todos los donantes del Banco de Sangre, Clínica Cardiovascular Santa María, Medellín, 1999.

\begin{tabular}{|c|c|c|c|c|c|c|}
\hline \multirow{2}{*}{ Variable Sexo } & \multicolumn{2}{|c|}{ Hombres } & \multicolumn{2}{|c|}{ Mujeres } & \multirow{2}{*}{$\begin{array}{l}\text { Kruskal } \\
\text { Wallis }\left(\chi^{2}\right)\end{array}$} & \multirow[b]{2}{*}{$\mathbf{p}^{*}$} \\
\hline & $\mathrm{X} \pm \mathrm{DE}$ & Varianza & $\mathrm{X} \pm \mathrm{DE}$ & Varianza & & \\
\hline Edad & $34(9,0)$ & 87,204 & $33(9,0)$ & 84,561 & 1,226 & 0,268 \\
\hline Presión sistólica & $126(15,0)$ & 227,093 & $117(14,0)$ & 194,268 & 257,989 & 0,000 \\
\hline Presión diastólica & $74(9,0)$ & 83,866 & $72(9,0)$ & 81,647 & 57,879 & 0,000 \\
\hline Peso & $73(11,0)$ & 118,605 & $62(9,0)$ & 86,359 & 792,267 & 0,000 \\
\hline Hemoglobina & $16,4(1,1)$ & 1,207 & $14,5(1,0)$ & 1,076 & 1366,821 & 0,000 \\
\hline
\end{tabular}

* Análisis no paramétrico

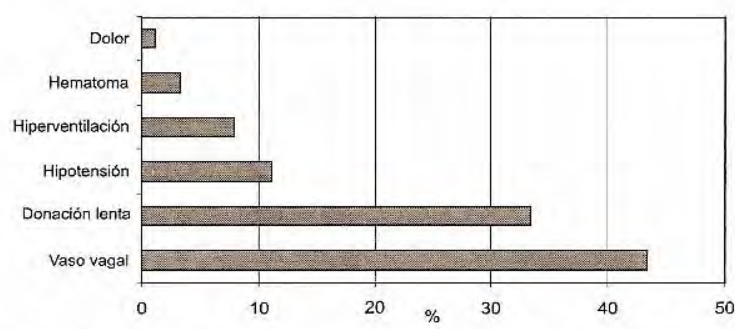

Figura 1. Distribución porcentual según tipo de reacción adversa en donantes del Banco de Sangre, Clínica Cardiovascular Santa María, Medellín.

Cuadro 2. Razón de disparidad, intervalos de confianza y nivel de significancia para los factores de riesgo estudiados en los donantes de la Clínica Cardiovascular Santa María, enero a diciembre de 1999.

\begin{tabular}{lccc}
\hline $\begin{array}{l}\text { Factor de } \\
\text { riesgo }\end{array}$ & $\begin{array}{c}\text { Razón de } \\
\text { disparidad }\end{array}$ & IC & $\boldsymbol{p}$ \\
\hline Sexo femenino & 2,43 & $1,54-3,86$ & 0,00001 \\
Peso $<55 \mathrm{~kg}$ & 1,81 & $0,98-3,31$ & 0,03000 \\
Edad $<25$ años & 2,82 & $1,77-4,48$ & 0,00000 \\
$\mathrm{Hb}<13 \mathrm{~g} / \mathrm{dl}$ & 0,36 & $0,02-2,44$ & 0,28890 \\
$\mathrm{PS} \leq 100 \mathrm{~mm} \mathrm{Hg}$ & 1,15 & $0,50-2,5$ & 0,71830 \\
$1^{\mathrm{a}}$ donación & 2,36 & $1,48-3,76$ & 0,00009 \\
$\mathrm{PD} \leq 60 \mathrm{~mm} \mathrm{Hg}$ & 1,15 & $0,55-2,33$ & 0,68000 \\
\hline
\end{tabular}

sicológico negativo para potenciales donantes, quienes podrían basarse en la experiencia de otros como argumento para rehusarse a donar sangre. Un mejor conocimiento de los posibles factores de riesgo para la aparición de efectos indeseables de la donación permite establecer medidas de prevención para disminuir el número de eventos; así mismo, aporta información fundamental para la conformación de grupos de donantes altruistas regulares.

Diversos factores relacionados con la experiencia de la donación influyen en el retorno de donantes a los bancos de sangre, entre ellos, el tratamiento recibido por parte del personal, el tiempo de espera para la atención, la habilidad técnica del funcionario y la presencia o no de malestar durante o después de la donación. Las instituciones que se dedican a la actividad de captar donantes de sangre deben hacer todos los esfuerzos para brindar información completa y atención óptima que garantice su bienestar.

En un estudio realizado por Thomson y colaboradores en una muestra de 34.726 donantes de cinco bancos de sangre de Estados Unidos, se encontró que el haber experimentado algún malestar durante o después de la donación era una variable de prediccón negativa para el retorno de donantes (5).

La frecuencia de reacciones adversas a la donación informadas en la literatura oscila entre 1 y $5 \%(2,6)$, similar a lo encontrado en el presente estudio, cuya frecuencia fue de $2,7 \%$.

La reacción vasovagal es el evento adverso más frecuente y se ha informado hasta en el 1,13\% de los donantes (2,7); en la presente investigación, ésta ocurrió en el 1,2\% de todos los donantes y representó casi la mitad $(43 \%)$ de todas las reacciones adversas.

Diferentes estudios han mostrado una mayor frecuencia de reacciones adversas en los donantes más jóvenes. Un estudio multicéntrico sobre reacciones vasovagales en 1.890 donantes que presentaron síncope, encontró que las variables más importantes en orden descendente eran la edad, el peso y la donación por primera vez (1).

Varios autores coinciden en que la edad menor de 25 a 30 años y la donación por primera vez son factores de riesgo para la presencia de efectos 
indeseables a la donación (1,2,6-8). Esta situación podría explicarse porque usualmente la primera donación se efectúa a más temprana edad; además, las personas jóvenes tienen sensibilidad barorreceptora ventricular más baja que los individuos mayores, quienes suelen ser hemodinámicamente más estables (1).

Por otro lado, se ha informado sobre diferencias individuales en la sensibilidad barorreceptora asociadas con la susceptibilidad a las reacciones vasovagales $(9,10)$. En un estudio se informó la relación inversa entre la historia familiar de hipertensión y la respuesta vasovagal durante la donación (9).

El sexo femenino fue un factor de predisposición a la reacción vasovagal, a diferencia de lo registrado en otros estudios $(1,6,7)$. Una posible explicación puede ser el peso corporal menor en el sexo femenino con relación al sexo masculino (cuadro 1), con la consiguiente hipovolemia, secundaria al mayor volumen de sangre perdido, en donantes de más baja estatura.

Muchos factores sicológicos y fisiológicos predisponen a la reacción vasovagal; para algunos autores, los aspectos sicológicos, así como la experiencia del personal del banco de sangre y los factores físicos son más importantes en la aparición de reacciones vasovagales que el sexo $(1,6,7)$.

Las reacciones adversas relacionadas con la venopunción (donación lenta, hematoma y dolor), aunque no generen pánico ni en los donantes ni el personal de servicio, también deben tenerse en cuenta, dado que pueden influir de forma negativa en la decisión de volver a donar.

En este estudio, la presencia de dolor o hematoma en el sitio de la punción representó menos del 1\% $(0,12 \%)$ de las reacciones en todos los donantes estudiados. Diferentes investigaciones realizadas en donantes informan frecuencias de estos tipos de reacción que oscilan entre 0,66 y $1,15 \%(11,12)$. Estos eventos son indicadores indirectos de la habilidad técnica y la competencia del personal del banco de sangre.
En conclusión, las reacciones adversas a la donación no son muy comunes, la reacción vasovagal es el evento más frecuente y los posibles factores de riesgo más importantes son la donación por primera vez, la edad menor de 25 años y el sexo femenino.

\section{Referencias}

1. Trouern-Trend JJ, Cable RG, Badon SJ, Newman BH, Popovsky MA. A case controlled multicenter study of vaso-vagal reactions in blood donors: influence of sex, age, donation status, weight, bloodpressure, and pulse. Transfusion 1999;39:316-20.

2. Popovsky MA, Whitaker B, Arnold NL. Severe outcomes of allogeneic and autologous blood donation frequency and characterization. Transfusion 1995; 35:734-7.

3. McCullough J. Blood donor medical assessment and blood collection. En: McCullough J, editor. Transfusion medicine. $1^{\text {st }}$ edition. New York: McGraw-Hill; 1998. p.4966.

4. Young J. Donor screening and phlebotomy. En: Rudmann $\mathrm{S}$, editor. Textbook of blood banking and transfusion medicine. $1^{\text {st }}$ edition. Philadelphia: W.B. Saunders Company; 1995. p.180-227.

5. Thomson RA, Bethel J, Lo AY, Ownby HE, Nass CC, Williams AE. Retention of "safe" blood donors. Transfusion 1998;38:359-67.

6. Tomasulo PA, Anderson AJ, Paluso MB, Gutschenritter MA, Aster RH. A study of criteria for blood donor deferral. Transfusion 1980;5:511-8.

7. Ogata $H$, linuma $N$, Nagashima $K$, Akabane $T$. Vasovagal reactions in blood donors. Transfusion 1980;6:67983.

8. Kwapisz MM, Dietrich G, Viehel H, Hempelmann H. Risk factors and frequency of adverse effects after autologous blood donation. Anaesthesist 1998;47:644-50.

9. Ditto B, Adler PS, France C, France J. Family history of hypertension and vaso-vagal symptoms during blood donation. J Behav Med 1995;18:331-40.

10. France $\mathbf{C}$. Baroreflex sensitivity during noxious stimulation in vaso-vagal reactors to blood donation. Int $\mathrm{J}$ Psychophysiol 1995;19:13-22.

11. Ranasinghe E, Harrison JF. Bruising following blood donation, its management and the response and subsequent return rates of affected donors. Transfus Med 2000;10:113-6.

12. McLeod BC, Price TH, Owen H, Ciavarella D, Snieccinski I, Randels MJ, et al. Frequency of immediate adverse effects associated with apheresis donation. Transfusion 1998;38:938-43. 